\title{
Tai Chi, Qigong and the Treatment of Depression and Anxiety
}

\author{
Robert W McGee* \\ Department of Graduate and Professional Studies in Business, Fayetteville State University, USA
}

*Corresponding author: Robert W McGee, Department of Graduate and Professional Studies in Business, Fayetteville

State University, USA

\section{ARTICLE INFO}

Received: May 25, 2021

Published: 幽 June 03, 2021

Citation: Robert W McGee. Tai Chi, Qigong and the Treatment of Depression and Anxiety. Biomed J Sci \& Tech Res 36(2)-2021. BJSTR. MS.ID.005823.

\section{ABSTRACT}

Qigong exercises are gentle exercises that have been a part of traditional Chinese medicine [TCM] for thousands of years. Tai chi is a series of gentle movements that improve balance, strength, flexibility and overall health. It incorporates qigong principles and is also a form of TCM. The present study summarizes the results of recent studies on the effect of qigong and tai chi on the treatment of depression.

Keywords: Depression; Anxiety; Qigong; Tai Chi; Taiji; Traditional Chinese Medicine; TCM; Baduanjin

\section{Introduction}

Qigong exercises have been a part of traditional Chinese medicine [TCM] for thousands of years $[1,2]$. They can be practiced individually or as a set of structured exercises as part of a health or fitness program. There are thousands of individual qigong exercises. Some of them are of a general nature, aimed at improving overall health, while others are of particular benefit to a certain organ or part of the body. Perhaps the most popular set of qigong exercises, both from a medical treatment perspective and as part of a general health regimen, is baduanjin, which consists of a series of eight exercises that improve general health while also stimulating all the major body organs and fascia [3-6]. It takes about 10-15 minutes to perform, depending on the speed and number of repetitions the individual chooses.

Several other sets of qigong exercises have become popular in recent years, both from a medical perspective and as part of a general health exercise program. As of this writing, the Chinese Health Qigong Association has published 9 DVD sets and instructional manuals that have been used by medical professionals as well as individuals to improve their health and the health of their patients [4,7-14]. Other publications have also discussed these series of exercises [15-20], as well as other qigong sets and the general theory of qigong [21-53]. Qigong is generally considered to be a subset of tai chi, although some scholars consider the two to be close cousins. Qigong is incorporated into the tai chi movements, but tai chi involves more than just qigong. Tai chi, also called taiji or taijiquan [54], is also a martial art, a gentle exercise routine, and an art as well as a sport. Both tai chi and qigong are forms of moving meditation. Although they are usually done from a standing position, certain routines and exercises can be performed from a seated position. Several styles of tai chi have evolved over the centuries, but the various styles have much in common. From a health perspective, the main styles all produce similar benefits because they all cause qi (pronounced chee) to flow and they all increase flexibility and strength.

Chen style tai chi is the oldest of the main styles. It originated in the Chen Village, China, probably in the thirteenth century [55]. The founder of the Yang style of tai chi was Yang Luchan, who studied the Chen style starting around 1820. He later became a teacher and is recognized as the founder of the Yang style, which is the most widely practiced style of tai chi today [56]. Sun-style tai chi is the youngest of the major styles of tai chi. It was founder by Sun Lutang around 1900 [57]. It is known for its high stances, which makes it suitable for elderly people who have balance issues. 
When tai chi is used as a tool of TCM, the Yang style is often chosen because it is gentler than the Chen style. The most universally practiced Yang-style form is the Yang-24, which consists of 24 different movements, some of which are repeated. It takes about 5-9 minutes to complete, depending on the speed with which the moves are done [58-60]. There are other Yang-style forms [61], most of which are longer than the Yang-24. Those forms a generally performed by practitioners as an art form, and in competitions, although it should be mentioned that the Yang-24 is also performed in competitions. Dr. Paul Lam has developed a set of Sun-style movements to aid in the treatment of arthritis $[62,63]$, although the same sequence of moves can also be used to treat other ailments. Although there are Sun-style forms that are used in competitions, the Sun style is not as popular as some other styles.

Tai chi and qigong have been used to treat and even prevent a wide range of ailments over the centuries [3,21-23]. The present article focuses on the use of tai chi and qigong to treat depression. In many cases, tai chi and/or qigong are used to treat other ailments, and one of the side benefits has been to reduce depression in patients who are suffering from depression as a result of those other ailments. In other words, many TCM practitioners do not prescribe the use of tai chi or qigong specifically for the treatment of depression (although they can). They prescribe their use for the treatment of other ailments, and have found that the patient's depression is reduced or eliminated as a result of practicing tai chi and/or qigong.

\section{Methodology}

The PubMed.gov database was used to find studies on tai chi, qigong and the treatment of depression [64].

\section{Findings}

A number of studies have found that the practice of tai chi and/or qigong have had a beneficial effect on patients who suffer from depression. Li et al. [65] examined the effect that mind-body exercises had on the mental health of patients suffering from Chronic Obstructive Pulmonary Disease (COPD). They examined the results of 13 randomized control trials (RCT) published between January, 1982 and June, 2019, which they used for meta-analysis. Those 13 studies used a variety of qigong and tai chi exercises, including baduanjin, wiqinxi, liuzijue, and both Yang and Sun style tai chi.

They found that mind-body exercises (tai chi, qigong and yoga) had significant benefits for COPD patients with anxiety ( $p=0.04$ ) and depression $(\mathrm{p}=0.000)$. Sub-group analysis found that $30-60$ minutes of exercise for 24 weeks of health qigong or yoga had a significant effect on anxiety for those over 70 who have had COPD for more than 10 years, and that health qigong sessions of 30 60 minutes, 2-3 times a week had a significant effect for patients suffering from depression who were over 70 years old and who had COPD for less than 10 years. They also found that the beneficial effects on long-term sufferers from COPD were less than those experienced by short-term sufferers of COPD.

Zou et al. [66] examined the results of randomized control trials (RCT) published between January, 1982 and October, 2017 for studies of the effect of baduanjin on depression and anxiety. Their focus was the effect on baduanjin on individuals with physical or mental illnesses. They found that the practice of baduanjin resulted in a significant decline in anxiety ( $\mathrm{p}<0.001)$ and depression ( $\mathrm{p}$ $<0.001$ ). Giménez-Meseguer et al. [67] reviewed studies on the effect of physical exercise and various mindfulness exercises on depression, anxiety and stress in patients with substance abuse problems. They found that physical exercise produced significantly better results when it came to stress reduction, but that tai chi and qigong produced significantly better results for reduction of depression and anxiety.

Many studies have found that the practice of qigong can have a positive effect on the reduction of depression. However, its underlying mechanism remains poorly understood. So et al. [68] conducted a study to systematically review and meta-analyze the existing literature on the use of qigong to reduce depression. They identified nine studies that covered both neurophysiological and psychological mechanisms. Five of the studies concluded that qigong was effective in reducing depression $(p<0.05)$, while four studies found no significant change. However, two studies were excluded in the meta-analysis due to insufficient information on the depression scores, and one of the insignificant studies had a $\mathrm{p}$ value of 0.053 , which indicates significance at the 10 percent level. They also found a significant effect on reducing diastolic blood pressure in some cases $(\mathrm{p}<0.05)$, while the effect on cortisol levels and systolic blood pressure were not significant. Their review concluded that qigong was effective in reducing depression through activating the parasympathetic nervous system.

Various qigong exercise sets were used in the studies they reviewed. Baduanjin was used in two of the studies, whereas seven other studies used seven different qigong sets - Yi Jin Ten-Section Brocades, Tai chi, Chan Ming Gong, self-healing qigong, Guolin New Qigong, Guolin Qigong, and Laughing Qigong. Liu et al. [69] studied the effects of tai chi on heart rate variability (HRV) in individuals age 60 or older with depression in Shanghai, China. Their study included 60 elderly individuals with depression scores of 10 or more on the Geriatric Depression Scale (GDS), who were randomly assigned to two groups of 30 each. Individuals in the experimental group practiced tai chi for 60 minutes, three times a week for 24 weeks. Those in the control group maintained their regular lifestyle. The experimental group practiced the Yang-24 form first, then progressed to the more complicated Yang-42 form after they had mastered the Yang-24 form. 
After 24 weeks, the tai chi group had significantly better scores for depression, and also had significantly better mean heart rate, RMSSD, HF, LFnorm and HFnorm scores ( $p<0.05$ ). These differences were not present in the control group. The researchers concluded that tai chi can be effective in reducing depression in older individuals. Zou et al. [70] studied the effects of meditative movements (tai chi, qigong and yoga) on major depressive disorder (MDD). They examined the meta-analysis of 15 randomized controlled trials (RCT) that used tai chi, qigong or yoga as intervention for MDD. Ethnicities varied, as did duration (4-12 weeks), session length (20-210 minutes) and the number of sessions per week (1 to 6).

They found that meditative movement had a significant effect on depression severity $(\mathrm{p}<0.001)$ and anxiety severity $(\mathrm{p}<$ 0.001 ). The use of meditative movement interventions resulted in significantly improved treatment remission rate $(\mathrm{p}<0.001)$ over passive controls. The study concluded that meditative movements for the treatment of MDD may provide a useful alternative to existing mainstream treatments such as drug therapy and psychotherapy. Osypuik et al. [71] explored the hypothesis that body postures in tai chi, qigong and related mind-body practices might be a biological factor that contributes to improvements in psychological wellbeing, the underlying premise being that posture affects mood. They found various studies that concluded that standing upright resulted in a better mood than being slumped over. The authors suggested an experimental approach to verifying their hypothesis.

Liu et al. [72] found that the practice of the Yang-24 tai chi form improved anxiety and depression and upregulates miR-1792 in coronary heart disease patients after percutaneous coronary intervention. The tai chi group practiced tai chi over an average of 10 months. At the end of that time, the changes in their anxiety subscale were significant ( $p=0.002)$, as were the changes in their depression subscale $(p=0.008)$. Luberto et al. [73] found that the practice of tai chi by patients with heart failure improved their depression symptoms and quality of life indexes. Lopez et al. [74] found that group tai chi and qigong classes taken by cancer patients and caregivers experienced significant improvement in global distress. Zhang et al. [75] performed a meta-analysis and evaluation of tai chi chuan on anxiety and depression on 14 experimental studies. They found that depression and anxiety were significantly reduced in both the younger and older adult groups, but that older adults benefitted more from tai chi than did younger adults.

Kong et al. [76] summarized a number of clinical trials that implemented tai chi as a treatment for depression. Their basic finding was that the practice of tai chi can relieve symptoms of depression. They also point out that the complexity of some tai chi movements might have an adverse effect on clinical use and suggest a simplified version might be more beneficial. They mention the Yang-8 form as one possibility. Its movements are easier to perform than some of the movements in the Yang-24 form, and the 8 -form does not take as long to complete as the 24-form. They also suggest customizing a tai chi regimen to fit different maladies, since different tai chi movements have differential effects on the various body organs. A regimen that might be appropriate for depression, for example, might be suboptimum for the treatment of certain other ailments.

Zhu et al. [77] examined the long-term effects of tai chi intervention on sleep and the mental health of females having dependence on amphetamine-type stimulants (ATS). The tai chi form used in their study was the Yang-24. Each session began with a 10-minute warmup, followed by 40 minutes of tai chi exercise, and ending with a 10-minute cooldown. They found that the practice of tai chi had positive effects on the quality of sleep, fitness and depression. They suggest that the practice of tai chi might be an inexpensive and potential supplementary treatment for ATSdependent individuals. These studies all seem to indicate that the practice of some form of tai chi or qigong can be beneficial in the treatment of depression and anxiety. Although the Yang-24 tai chi form and the baduanjin qigong set seem to be the most frequently used forms used in these studies, they are not the only forms. Regardless of which set of forms is chosen, qi (energy) is generated and assists in the alleviation of anxiety and depression.

\section{References}

1. (2021) Wikipedia. History of Qigong.

2. Douglas Bill (1999) The Complete Idiot's Guide to T'ai Chi \& QiGong. New York: Alpha Books, a division of Macmillan General Reference.

3. McGee, Robert W (2020) Ba Duan Jin as a Treatment for Physical Ailments: A Bibliography of Recent Medical Research, Fayetteville State University, Broadwell College of Business and Economics, Studies in the Economics of Qigong.

4. (2008) Chinese Health Qigong Association. Ba Duan Jin. Beijing: Foreign Languages Press.

5. Lu Yan (2011) Experimental Research of Expanding the Spare Exercises with Ba Duan Jin in the Group of Graduates - Take the Graduates of ECNU for Example. Master's Thesis. East China Normal University.

6. (2021) Wikipedia. Baduanjin qigong.

7. (2007) Chinese Health Qigong Association. Liu Zi Jue. Beijing: Foreign Languages Press.

8. (2008) Chinese Health Qigong Association. Wu Qin Xi. Beijing: Foreign Languages Press.

9. (2009) Chinese Health Qigong Association. Yi Jin Jing. Beijing: Foreign Languages Press.

10. (2014) Chinese Health Qigong Association. Da Wu. Beijing: Foreign Languages Press.

11. (2014) Chinese Health Qigong Association. Shi Er Duan Jin. Beijing: Foreign Languages Press.

12. (2014) Chinese Health Qigong Association. 2014. Daoyin Yangsheng Gong Shi Er Fa. Beijing: Foreign Languages Press.

13. (2014) Chinese Health Qigong Association. 2014. Mawangdui Daoyin Shu. Beijing: Foreign Languages Press. 
14. (2014) Chinese Health Qigong Association. 2014. Taiji Yangsheng Zhang. Beijing: Foreign Languages Press.

15. (2021) Wikipedia. Liu Zi Jue.

16. (2021) Wikipedia. Five Animals.

17. McGee, Robert W (2020) Wu Qin Xi as a Treatment for Physical Ailments: A Bibliography of Recent Medical Research. Fayetteville State University, Broadwell College of Business and Economics, Studies in the Economics of Qigong.

18. (2021) Wikipedia Yi Jin Jing.

19. McGee, Robert W (2020) The Use of Yi Jin Jing to Treat Illness: A Summary of Three Studies, Fayetteville State University, Broadwell College of Business and Economics, Studies in the Economics of Qigong No 5, August 31.

20. McGee, Robert W (2021) The Use of Yi Jin Jing to Treat Illness: A Summary of Three Studies. Academia Letters, Article 547.

21. McGee, Robert W (2020) Qigong: A Bibliography of Books and Other Materials, Fayetteville State University, Broadwell College of Business and Economics, Studies in the Economics of Qigong, No. 1, August 25.

22. McGee, Robert W (2020) A Bibliography of Recent Medical Research on Qigong, Fayetteville State University, Broadwell College of Business and Economics, Studies in the Economics of Qigong, No. 2, August 31.

23. McGee, Robert W (2020) Qigong and the Treatment and Prevention of COVID-19, Fayetteville State University, Broadwell College of Business and Economics, Studies in the Economics of Qigong, No. 6, September 4.

24. McGee, Robert W (2021) Tai Chi, Qigong and Transgender Health. Fayetteville State University, Broadwell College of Business and Economics, Studies in the Economics of Tai Chi and Qigong, No. 8, April 16.

25. McGee Robert W (2021) Tai Chi, Qigong and the Treatment of Disease. Biomed J Sci \& Tech Res 34(2).

26. McGee, Robert W (2021) Tai Chi, Qigong and the Treatment of Cancer. Biomed J Sci \& Tech Res 34(5).

27. Frantzis Bruce (2005) Opening the Energy Gates of Your Body: Qigong for Lifelong Health. Blue Snake Books.

28. Frantzis Bruce (2010) Dragon and Tiger Medical Qigong, Volume 1. Fairfax, CA: Energy Arts.

29. Frantzis, Bruce (2014) Dragon and Tiger Medical Qigong Volume 2: Qi Cultivation Principles and Exercises. North Atlantic Books.

30. Jahnke Roger (1997) The Healer Within. San Francisco: Harper.

31. Jahnke Roger (2002) The Healing Promise of Qi. New York: Contemporary Books, a division of McGraw-Hill.

32. Ce Jin, Hu Zhanggui, Jin Zhenghua (1996) Practical Chinese Qigong for Home Health Care. Beijing: Foreign Languages Press.

33. Friedman, Suzanne (2006) Medical Qigong Exercise Prescriptions: A Self-Healing Guide for Patients \& Practitioners. Xlibris.

34. Johnson, Jerry Alan (2000) Chinese Medical Qigong Therapy: A Comprehensive Clinical Guide. Pacific Grove, CA: International Institute of Medical Qigong.

35. Johnson, Jerry Alan (2005) Chinese Medical Qigong Therapy Vol. 1: Energetic Anatomy and Physiology. Pacific Grove, CA: International Copyright@ Robert W McGee | Biomed J Sci \& Tech Res | BJSTR. MS.ID.005621. Volume 34- Issue 5 DOI: 10.26717/ BJSTR.2021.34.005621 27177 Institute of Medical Qigong.

36. Johnson, Jerry Alan (2005) Chinese Medical Qigong Therapy, Vol. 2: Pacific Grove, CA: International Institute of Medical Qigong.

37. Johnson, Jerry Alan (2002) Chinese Medical Qigong Therapy, Vol. 3: Pacific Grove, CA: International Institute of Medical Qigong.
38. Johnson, Jerry Alan (2002) Chinese Medical Qigong Therapy, Vol. 4: Prescription Exercises and Meditations, Treatment of Internal Diseases, Pediatrics, Geriatrics, Gynecology, Neurology, and Energetic Psychology. Pacific Grove, CA: International Institute of Medical Qigong.

39. Johnson, Jerry Alan (2005) Chinese Medical Qigong Therapy, Vol. 5: An Energetic Approach to Oncology. Pacific Grove, CA: International Institute of Medical Qigong.

40. Liu, Tianjun, Xiao Mei Qiang (2013) Chinese Medical Qigong. London \& Philadelphia: Singing Dragon.

41. Moon Sanghee (2019) Effects of a Mind-Body Exercise on Non-motor Symptoms and Inflammatory Biomarkers in Parkinson's Disease. PhD dissertation. University of Kansas.

42. Morris, Kim Lisa Chou (1998) On the Chinese Spiritual Tradition of Qigong in Toronto as a Site for Negotiating Identity. Master of Fine Arts Thesis. York University (Canada).

43. Murakawa, Haruhiko (2002) Phenomenology of the Experience of Qigong: A Preliminary Research Design for the Intentional Bodily Practices. PhD dissertation. California Institute of Integral Studies.

44. Tapajna, Mark (2006) An Examination of Medical Qigong: Its Practice, Application, and Potential Value for a Western Society. PhD dissertation. Cleveland State University.

45. Chau E (2014) Guolin Qigong, Natural Walk, Walking Qigong, AntiCancer Qigong.

46. Rosenberg D (2016) Introduction to Guo Lin Chi Kung.

47. Rosenberg D (2016) Guo Lin Chi Kung - Opening Exercises.

48. Rosenberg D (2016) Guo Lin Chi Kung - beginning walking, the basic walking style.

49. Rosenberg, D (2016) Guo Lin Chi Kung - three part gathering exercises.

50. Rosenberg D (2016) Guo Ling Chi Kung - other walking styles.

51. Rosenberg D (2016) Guo Lin Chi Kung - medium fast and quick walking styles.

52. Rosenberg D (2016) Guo Lin Chi Kung - Fundamentals of Practice

53. YK Mak (2012) 18 Forms Tai Chi Qigong. Wan Li Book Co. Hong Kong. $7^{\text {th }}($ Edn.).

54. (2021) Wikipedia. Tai Chi.

55. (2021) Wikipedia. Chen-style taijiquan.

56. (2021) Wikipedia. Yang-style t'ai chi ch'uan.

57. (2021) Wikipedia. Sun-style t'ai chi ch'uan.

58. (2021) Wikipedia. 24-form tai chi chuan.

59. (2021) 24 Form Tai Chi Demonstration Back View Master Amin Wu, 24 Form Tai Chi Demonstration Back View Master Amin Wu.

60. (2021) 24 Step Simplified Tai Ji Quan by Master Faye Yip.

61. (2021) Tai Chi and Qigong-Yang Style Tai Chi.

62. (2021) Tai Chi for Health Institute.

63. (2021) Tai Chi for Arthritis Video. Dr. Paul Lam. Free Lesson and Introduction. Tai Chi for Health Institute.

64. (2021) PubMed.gov. National Library of Medicine, National Center for Biotechnology Information, National Institute of Health.

65. Li Z, Liu S, Wang L, Smith L (2019) Mind-Body Exercise for Anxiety and Depression in COPD Patients: A Systematic Review and Meta-Analysis. International journal of environmental research and public health 17(1): 22 . 
66. Zou L, Yeung A, Quan X, Hui SS, Hu X, et al. (2018) Mindfulness-Based Baduanjin Exercise for Depression and Anxiety in People with Physical or Mental Illnesses: A Systematic Review and Meta-Analysis. International journal of environmental research and public health, 15(2): 321.

67. Giménez-Meseguer J, Tortosa-Martínez J, Cortell-Tormo JM (2020) The Benefits of Physical Exercise on Mental Disorders and Quality of Life in Substance Use Disorders Patients. Systematic Review and MetaAnalysis. International journal of environmental research and public health 17(10): 3680 .

68. So W, Cai S, Yau SY, Tsang H (2019) The Neurophysiological and Psychological Mechanisms of Qigong as a Treatment for Depression: A Systematic Review and Meta-Analysis. Frontiers in psychiatry 10: 820.

69. Liu J, Xie H, Liu M, Wang Z, Zou L, et al. (2018) The Effects of Tai Chi on Heart Rate Variability in Older Chinese Individuals with Depression. International journal of environmental research and public health, 15(12): 2771

70. Zou L, Yeung A, Li C, Wei GX, Chen KW, et al. (2018) Effects of Meditative Movements on Major Depressive Disorder: A Systematic Review and Meta-Analysis of Randomized Controlled Trials. Journal of clinical medicine 7(8): 195.

71. Osypiuk K, Thompson E, Wayne PM (2018) Can Tai Chi and Qigong Postures Shape Our Mood? Toward an Embodied Cognition Framework for Mind-Body Research. Frontiers in human neuroscience 12: 174.

\section{ISSN: 2574-1241}

DOI: 10.26717/BJSTR.2021.36.005823

Robert W McGee. Biomed J Sci \& Tech Res

This work is licensed under Creative Commons Attribution 4.0 License

Submission Link: https://biomedres.us/submit-manuscript.php
72. Liu J, Yu P, Lv W, Wang X (2020) The 24-Form Tai Chi Improves Anxiety and Depression and Upregulates miR-17-92 in Coronary Heart Disease Patients After Percutaneous Coronary Intervention. Frontiers in physiology 11: 149.

73. Luberto CM, Coey CA, Davis RB, Wayne PM, Crute S, et al. (2020) Exploring correlates of improved depression symptoms and quality of life following tai chi exercise for patients with heart failure. ESC heart failure 7(6): 4206-4212.

74. Lopez G, Narayanan S, Christie A, Powers-James C, Liu W, et al. (2020) Effects of Center-Based Delivery of Tai Chi and Qi Gong Group Classes on Self-Reported Symptoms in Cancer Patients and Caregivers. Integrative cancer therapies 19: 1534735420941605.

75. Zhang S, Zou L, Chen LZ, Yao Y, Loprinzi PD, et al. (2019) The Effect of Tai Chi Chuan on Negative Emotions in Non-Clinical Populations: A MetaAnalysis and Systematic Review. International journal of environmental research and public health 16(17): 3033.

76. Kong J, Wilson G, Park J, Pereira K, Walpole C, et al. (2019) Treating Depression with Tai Chi: State of the Art and Future Perspectives. Frontiers in psychiatry 10: 237.

77. Zhu D, Dai G, Xu D, Xu X, Geng J, et al. (2018) Long-Term Effects of Tai Chi Intervention on Sleep and Mental Health of Female Individuals with Dependence on Amphetamine-Type Stimulants. Frontiers in psychology 9: 1476 .

$\begin{array}{ll}\text { BIOMEDICAL } & \text { Assets of Publishing with us } \\ \text { RESEARCHES } & \text { - Global archiving of articles } \\ \text { - Immediate, unrestricted online access }\end{array}$

